\title{
Efeito de diferentes profundidades de cama sobre parâmetros ambientais para suínos em crescimento e terminação
}

\author{
Érico K. Corrêa ${ }^{1,4}$, Thomaz Lucia Jr. ${ }^{2}$, Carlos Gil-Turnes ${ }^{2}$, Marcio N. Corrêa ${ }^{2}$, Ivan Bianchii ${ }^{1}$, José L. Corezzolla ${ }^{3}$ \& Rafael R. Ulguim ${ }^{3}$
}

\section{RESU M O}

A suinocultura, embora considerada atividade de alto potencial poluidor em virtude da grande concentração de animais e ao volume de dejetos líquidos produzidos por unidade de área, enfrenta um grande desafio que se refere ao desenvolvimento de sistemas alternativos para a produção de suínos, que diminuam o potencial poluidor desta atividade. Pela potencialidade de absorver o esterco e a urina dos suínos, o sistema de cama pode ser uma alternativa viável para o manejo dos efluentes, mas este sistema apresenta aspectos negativos para o conforto térmico dos suínos. 0 bjetivou-se, com este trabalho, avaliar o efei to de diferentes alturas de cama com casca de arroz, utilizada no sistema de produção sobre cama durante as fases de crescimento e terminação, sobre indicadores de condicionamento ambiental da edificação zootécnica (temperatura ambiente e da cama e umidade relativa do ar). Três tratamentos foram comparados (duas profundidades diferentes de cama de casca de arroz, 0,5 e 0,25 m, e um sistema com piso compacto de concreto). A utilização de piso com cama para suínos nas fases de crescimento-terminação, mesmo com diferentes profundidades, não foi suficiente para alterar os indicadores de condicionamento ambiental da edificação zootécnica.

Palavras-chave: meio ambiente, dejetos, conforto térmico, compostagem

\section{Effect of different bedding depths on ambiental parameters for growing and finishing swine}

\begin{abstract}
Due to the large animal inventories and the great volume of waste, swine production is considered a highly polluting activity. Therefore one of its greatest challenges is the development of alternative production systems with lower polluting potential. Deep litter systems may be feasible alternative for waste management due to their capacity for slurry absorption, although they may negatively influence the thermal comfort of the pigs. The objective of this study was to evaluate the effect of distinct depths of rice husk litters used to raise pigs in growing and finishing phases on ambiental temperature and relative humidity inside the barn. Three treatments were compared: litter with a depth of $0.5 \mathrm{~m}$; litter with a depth of $0.25 \mathrm{~m}$; and solid concrete floor. Ambiental temperature and relative humidity inside the barn were not influenced by the use of litter or by different litter depths.
\end{abstract}

Key words: environment, waste, thermal comfort, composting

\footnotetext{
${ }_{1}^{1}$ Centro de Biotecnologia Agrícola, UFPel, CP 354, CEP 96010-900, Pelotas, RS. Fone/Fax (53) 3275-7588. E-mail: ekcorrea@ufpel.edu.br

2 Faculdade de Veterinária/UFPel. Fone/Fax (53) 3275-7290. E-mail: thomaz@ufpel.edu.br

3 Graduando em M edicina Veterinária/UFPel. Fone/Fax (53) 3275-7588. E-mail: rulguim_fv@ufpel.edu.br

${ }^{4}$ Bolsista CN Pq
} 


\section{INTRODUÇÃO}

Como resultado da transformação intensiva de nutrientes de baixo valor agregado (milho e soja) por meio da produção de suínos, são obtidos alimentos de alto valor; porém ocorre a geração de grande quantidade de dejetos por unidade de área que, quando não tratados adequadamente, constituem alto risco ao meio ambiente (Wang et al., 2004). Os investimentos para o tratamento de efluentes líquidos, por outro lado, nem sempre são compatíveis com a realidade econômica dos criadores e representam importante barreira para a solução do problema (Corrêa et al., 2000; Honeyman, 2005).

A estrutura da produção de suínos no Brasil mudou drasticamente nas últimas décadas, atualmente, no entanto, as matérias-primas utilizadas na alimentação dos animais são produzidas em regiões diferentes daquelas onde os suínos são criados (Bartels, 2001); deste modo, nas áreas de grande concentração de suínos se produzem dejetos em quantidade superior à capacidade de absorção do solo, extrapolando a recomendação técnica de sua utilização como adubo, provocando a degradação ambiental (Jongbloed \& Lenis, 1998, Gentry et al., 2002).

Sistemas alternativos para a produção de suínos têm despertado o interesse do setor produtivo, principalmente por apresentarem, quando comparados aos sistemas convencionais, edificações de menor custo, melhora do bem-estar dos animais e menor impacto ao meio ambiente (Honeyman, 1996); assim, o sistema de produção de suínos sobre cama (SPC) vem ganhando espaço junto aos produtores, sobretudo por facilitar e reduzir os custos com o manejo dos dejetos, quando comparado com o sistema convencional de produção (Honeyman \& Harmon, 2003).

Outro aspecto favorável do SPC, é que este sistema possibilita a comercialização da produção em determinados nichos de mercado, como o de produtos orgânicos que, via de regra, permite que o produtor receba uma remuneração melhor no momento de negociar os animais (Fraser, 2001).

O SPC tem, como princípio, a substituição do piso convencional (concreto, ferro ou plástico), por uma cama de 50 $\mathrm{cm}$ de profundidade com material rico em carbono (serragem, casca de arroz, talos de milho ou palha de cereais) (Oliveira et al., 1999; Corrêa et al., 2000; Gentry et al., 2002). Esta camada desempenha a dupla função de piso e digestor dos dejetos, que são retidos, armazenados e estabilizados dentro da própria edificação suinícola e manejados em estado sólido.

O processo de estabilização dos dejetos que ocorre no interior da cama é semelhante ao da compostagem (Oliveira et al., 1999), porém nesta não se dá a adição de material após seu início, mas no SPC ela ocorre diariamente (água e ração desperdiçada pelos animais, fezes e urina); enquanto os dejetos líquidos apresentam menos de $10 \%$ de matéria seca, os dejetos originários da cama indicam aproximadamente $40 \%$ de matéria seca (Bartels, 2001).

$\mathrm{Na}$ medida em que acontece o aumento do conteúdo de matéria seca dos dejetos ocorre, também, o aumento da concentração de nutrientes, tornando os dejetos mais valoriza- dos, do ponto de vista agronômico (Wang et al., 2004; Honeyman, 2005). Esta concentração de nutrientes se deve principalmente à evaporação da fração líquida, que é absorvida temporariamente pela cama. O calor necessário para incrementar a concentração dos nutrientes é obtido, em parte, durante a fase termofílica da compostagem; nesta etapa do processo de estabilização dos dejetos, a temperatura no interior da cama atinge valores superiores a $40{ }^{\circ} \mathrm{C}$ durante mais de 90 dias (Corrêa et al., 2000; Tiquia, 2005).

A produção de suínos em SPC apresenta a desvantagem de, em épocas ou locais de clima quente, prejudicar o conforto ambiental dos animais extrapolando, durante a fase termofílica, o limiar de temperatura recomendado para os suínos em terminação, devido aos processos que ocorrem durante a compostagem in situ dos dejetos da criação (Corrêa et al., 2000). O processo de compostagem se inicia a temperatura ambiente predominando, nesta fase, microrganismos mesófilos. Na medida em que as ações dos microrganismos se intensificam, ocorrem reações exotérmicas em virtude da decomposição da fração leve da matéria orgânica; alguns dias depois, a temperatura aumenta gradativamente podendo atingir valores superiores a $65^{\circ} \mathrm{C}$, quando prevalecem microrganismos termófilos (Bartels, 2001; Kapuinen, 2001; Tang et al., 2004); deste modo, camas com diferentes alturas poderiam diferir em temperatura durante a fase termofílica, propiciando diferentes condições ambientais no interior da edificação.

Objetivou-se, com este trabalho, avaliar o efeito de diferentes alturas de cama com casca de arroz utilizadas no SPC, nas fases de crescimento e terminação, sobre indicadores de condicionamento ambiental (temperatura na superfície da cama, temperatura ambiente e umidade relativa do ar) da edificação zootécnica.

\section{MATERIAL E MÉTODOS}

O experimento foi conduzido no Centro Agropecuário da Palma, da Universidade Federal de Pelotas, localizado no município de Capão do Leão, RS, Brasil (Latitude - 31 $45^{\circ}$ $\mathrm{S}$ - Longitude - $52^{\circ} 21^{\prime} \mathrm{W}$ ), no período de julho de 2003 a julho de 2004. Compararam-se três tratamentos (3 baias), constituídos de duas profundidades diferentes de cama de casca de arroz, 0,5 m (T1) e 0,25 m (T2), e um sistema controle com piso compacto de concreto (T3); as baias, que receberam T1 e T2, também possuíam piso de concreto sob a cama.

Cada baia com 5 animais foi adotada como unidade experimental e cada tratamento apresentou quatro repetições, a primeira de julho a setembro de 2003, a segunda de outubro a dezembro de 2003, a terceira de fevereiro a abril de 2004 e a última de maio a julho de 2004, havendo, portanto, substituição total das camas entre a segunda e a terceira repetição.

As baias utilizadas foram dispostas lado a lado, com 7,0 $\mathrm{m}^{2}$ cada uma $(2,0 \times 3,50 \mathrm{~m})$, e área de $1,4 \mathrm{~m}^{2}$ por suíno. A cama foi disponibilizada no volume de $0,7 \mathrm{~m}^{3}$ no $\mathrm{T} 1$ e 0,35 $\mathrm{m}^{3}$ no $\mathrm{T} 2$ por animal, sendo a casca de arroz distribuída em 
toda a área das baias que receberam os tratamentos com cama ( 0,50 ou $0,25 \mathrm{~m}$ de profundidade); cada cama foi utilizada em duas repetições, sem adição de material complementar mas com revolvimento (aeração) entre as repetições utilizando-se escarificador manual. A edificação tinha cobertura de telha de barro, com $3,2 \mathrm{~m}$ de pé direito e divisórias internas e externas de alvenaria; outrossim, cada baia possuía um comedouro convencional de três bocas e um bebedouro tipo nipple.

Foram avaliados 60 animais $F_{1}$ (Landrace $\mathrm{x}$ Large White), sendo 15 animais por repetição, com 5 em cada baia (2 machos castrados e 3 fêmeas); a avaliação ocorreu dos 60 aos 145 dias (42 dias de crescimento e 45 de terminação), obedecendo o delineamento experimental inteiramente casualizado. Os animais foram alimentados ad libitum com uma ração composta de $19 \%$ de proteína bruta e $3.350 \mathrm{kcal}$ de $\mathrm{EM} \mathrm{kg}{ }^{-1}$ na fase de crescimento e $17 \%$ de proteína bruta e $3.200 \mathrm{kcal}^{\mathrm{N}} \mathrm{EM} \mathrm{kg}{ }^{-1}$ na fase de terminação (NRC, 1998).

Determinaram-se as medidas de condicionamento ambiental da edificação por meio do registro das temperaturas com uso de termômetro digital com sonda Multi-Stem ${ }^{\circ}\left(-50\right.$ a $150{ }^{\circ} \mathrm{C}$, $\pm 1{ }^{\circ} \mathrm{C}$ ). Registrou-se a temperatura das camas na superfície no centro (TSC) e na superfície na frente (TSF) das baias e a meia profundidade, tanto no centro (TMC) como na frente (TMF) das baias. Para o T1, registrou-se também a temperatura da cama a $45 \mathrm{~cm}$ de profundidade no centro (T45C) e a $45 \mathrm{~cm}$ de profundidade na frente (T45F) da baia, além da temperatura na superfície do piso para o T3. A temperatura ambiente (TA) e a umidade relativa do ar (UR) foram medidas em cada tratamento utilizando-se higrômetro de bulbo úmido e bulbo seco, instalado a $70 \mathrm{~cm}$ de altura do piso. Todas as medidas foram realizadas semanalmente, às 9 e $15 \mathrm{~h}$.

Os efeitos dos tratamentos, repetições de cada mês do período experimental e de potenciais interações entre esses fatores, foram avaliados através de análise de variância, com comparação entre médias, por meio do teste de Tukey. Contrastes ortogonais foram utilizados para comparações entre camas novas ( $\mathrm{CN}$ - primeira e terceira repetições) e velhas (CV - segunda e quarta repetições); primeira (PC - primeira e segunda repetições) e segunda cama (SC - terceira e quarta repetições). As comparações entre médias para os contrastes foram realizadas pelo teste de Schefeé, assim como todas as avaliações foram realizadas com o software Statistix (2003).

\section{RESULTADOS E DISCUSSÃO}

Não ocorreu efeito de tratamento para as variáveis UR e TA nos diferentes tratamentos (Tabela 1); quanto às TSC e TSF, os valores registrados para o T3 foram inferiores aos demais tratamentos $(\mathrm{P}<0,05)$, porém, o efeito das diferentes alturas de cama não foi suficiente para alterar a resposta para TSC e TSF entre os tratamentos T1 e T2 (P > 0,05). Notou-se efeito dos tratamentos sobre a TMC e TMF $(\mathrm{P}<0,05)$, sendo a temperatura média superior no $\mathrm{T} 1$ em relação ao $\mathrm{T} 2$.
Tabela 1. Médias ${ }^{1}$ de umidade relativa do ar (\%), temperatura ambiental e das camas $\left({ }^{\circ} \mathrm{C}\right)$ em diferentes locais e profundidades por tratamento, repetição e mês

\begin{tabular}{|c|c|c|c|c|c|c|c|c|}
\hline & UR & TA & TSC & TMC & T45C & TSF & TMF & T45F \\
\hline \multicolumn{9}{|c|}{ Tratamento } \\
\hline 1 & $81,2 \mathrm{a}$ & $18,3 \mathrm{a}$ & $26,1 \mathrm{a}$ & $35,8 \mathrm{a}$ & 30,9 & $24,2 \mathrm{a}$ & $30,6 a$ & 27,6 \\
\hline 2 & $81,4 \mathrm{a}$ & $18,2 a$ & $24,6 \mathrm{a}$ & $32,1 b$ & - & $23,2 a$ & $27,7 b$ & - \\
\hline 3 & $80,6 \mathrm{a}$ & $17,7 \mathrm{a}$ & $17,8 \mathrm{~b}$ & - & - & $17,9 \mathrm{~b}$ & - & - \\
\hline \multicolumn{9}{|c|}{ Repetição } \\
\hline 1 & $80,7 \mathrm{a}$ & $15,9 \mathrm{~b}$ & $25,0 \mathrm{a}$ & $40,8 \mathrm{a}$ & $32,4 a$ & $21,4 \mathrm{~b}$ & $27,5 b$ & $25,4 b$ \\
\hline 2 & $79,8 \mathrm{~b}$ & $20,0 \mathrm{a}$ & $22,8 b$ & $30,9 b$ & $28,6 \mathrm{~b}$ & $22,2 b$ & $29,1 b$ & $26,7 b$ \\
\hline 3 & $79,6 \mathrm{~b}$ & $20,3 a$ & $24,5 b$ & $38,8 \mathrm{a}$ & $36,2 \mathrm{a}$ & $24,8 \mathrm{a}$ & $35,6 a$ & 34,1 a \\
\hline 4 & $84,2 \mathrm{a}$ & $16,2 \mathrm{~b}$ & $19,1 \mathrm{C}$ & $25,3 \mathrm{c}$ & $26,4 \mathrm{c}$ & $18,5 \mathrm{C}$ & $24,3 \mathrm{C}$ & $24,3 b$ \\
\hline \multicolumn{9}{|c|}{ Mês } \\
\hline 1 & $80,4 a$ & $19,2 \mathrm{a}$ & $22,1 b$ & $31,3 b$ & $30,9 b$ & $22,3 \mathrm{a}$ & $29,6 a$ & $28,9 a$ \\
\hline 2 & $81,0 \mathrm{a}$ & $17,9 \mathrm{~b}$ & $24,2 \mathrm{a}$ & $39,9 a$ & $33,8 \mathrm{a}$ & $22,0 \mathrm{a}$ & $31,0 \mathrm{a}$ & $29,3 a$ \\
\hline 3 & $81,8 \mathrm{a}$ & $17,2 \mathrm{~b}$ & $22,2 b$ & $30,7 b$ & $28,1 \mathrm{~b}$ & $20,9 a$ & $26,7 \mathrm{~b}$ & $24,7 \mathrm{~b}$ \\
\hline
\end{tabular}

${ }^{1}$ Médias seguidas da mesma letra na coluna não diferem significativamente $(\mathrm{P}<0,05)$. UR - umidade relativa do ar, TA - temperatura ambiente, TSC temperatura da cama na superfície, no centro da baia, TMC - temperatura da cama a meia profundidade, no centro da baia, T45C - temperatura da cama a $45 \mathrm{~cm}$ de profundidade, no centro da baia, TSF - temperatura da cama na superfície, na frente da baia, TMF - temperatura da cama a meia profundidade, na frente da baia, $\mathrm{T} 45 \mathrm{~F}$ - temperatura da cama a $45 \mathrm{~cm}$ de profundidade, na frente da baia

A presença de cama, mesmo em diferentes alturas dentro da baia, não foi suficiente para alterar a resposta da variável UR. Em todos os tratamentos a UR foi superior à recomendada por Benedi (2002) de $70 \%$ e por Veit \& Troutt (1999) de 75\% para suínos em crescimento e terminação. De acordo com Morrison et al. (1969), valores elevados de UR diminuem a perda de calor dos suínos por evaporação, principalmente pelos pulmões, afetando o desempenho zootécnico dos animais; com relação a TA, esta não foi influenciada pelos sistemas de piso e mesmo o calor gerado nas camas devido à fase termofílica (Kapuinen, 2001), não foi suficiente para modificar os valores observados para TA. Os valores registrados para temperatura ambiente nos diferentes tratamentos se situaram dentro do intervalo recomendado por Veit \& Troutt (1999), para suínos em crescimento e terminação de 12 a $21{ }^{\circ} \mathrm{C}$, mas T1 e T2 mostraram valores médios ligeiramente superiores ao recomendado por Benedi (2002) de 15 a $18{ }^{\circ} \mathrm{C}$.

$\mathrm{O}$ valor médio observado para a variável temperatura na superfície dos pisos foi inferior para o T3 em relação aos demais devido, seguramente, a temperatura originada no interior da cama ter sido suficiente para alterar a temperatura na superfície (Venglovsky et al., 2005); entretanto, as diferenças nas alturas de cama não foram suficientes para provocar diferenças entre os T1 e T2; a diferença das camas para o piso de concreto pode interferir no comportamento dos suínos visto que animais em terminação passam, em média, de 60 a $80 \%$ do tempo deitados, e a superfície em contato 
com o piso representa de 10 a $20 \%$ da superfície corporal total (Oliveira et al., 1999).

A temperatura média a meia profundidade $(15 \mathrm{~cm})$ das camas foi superior no $\mathrm{T} 1$ quando comparada à do T2, podendo indicar maior atividade microbiana durante a fase termofílica (Tang et al., 2004), devido à maior altura de cama, já que a temperatura da cama possui correlação positiva com a atividade microbiana (Tiquia, 2005), resultando em uma temperatura média mais elevada no T1.

Observou-se, por meio da Tabela 1, que ocorreu efeito de repetição sobre a UR $(P<0,05)$; a UR foi superior na quarta repetição mas a primeira repetição não diferiu das demais. Os maiores valores de TA foram observados nas segunda e terceira repetições $(\mathrm{P}<0,05)$, coincidindo com as estações mais quentes do ano. Os maiores valores para TSC foram constatados para as primeira e terceira repetições $(\mathrm{P}<0,05)$, porém esta última não diferiu da segunda repetição $(\mathrm{P}>$ 0,05); para TSF, o maior valor foi captado na terceira repetição, enquanto a menor média observada para TSC e TSF foi na quarta repetição $(\mathrm{P}<0,05)$; as médias observadas para TMC nas primeira e terceira repetições foram superiores às demais $(\mathrm{P}<0,05)$ e, para TMF, o maior valor registrado foi na terceira repetição $(\mathrm{P}<0,05)$; enfim, tanto a TMC quanto a TMF mostraram a menor média na quarta repetição.

O maior valor médio para umidade relativa do ar ao longo dos lotes consecutivos, foi registrado na quarta repetição, provavelmente como reflexo das condições externas, visto que as edificações adotadas neste estudo possuíam laterais abertas; apesar disto, em todas as repetições as médias de umidade relativa do ar ficaram situadas acima dos $70 \%$ recomendados por Benedi (2002) e de 75\%, por Veit \& Troutt (1999), para suínos em fase de crescimento e terminação.

Os maiores valores de TA foram registrados nas épocas quentes do ano (segunda e terceira repetições), mesmo que em todos os lotes os valores tenham permanecido dentro da faixa de 12 a $21{ }^{\circ} \mathrm{C}$, sugerida por Veit \& Troutt (1999); nas épocas quentes, porém, os valores registrados superaram a faixa de 15 a $18{ }^{\circ} \mathrm{C}$ indicada por Benedi (2002) para suínos em crescimento e terminação; ressalta-se que em repetições realizadas em épocas quentes, o desempenho produtivo dos animais pode ter sido prejudicado, visto que animais em crescimento e terminação são mais sensíveis a altas temperaturas (Corrêa et al., 2000; Rinaldo et al., 2000).

Com respeito às variáveis TSC e TMC, os maiores valores foram observados nas primeira e terceira repetições, quando as camas eram novas e, conseqüentemente, estavam na fase termofílica, com grande produção de calor (Tiquia et al., 1997; Venglovsky et al., 2005). A temperatura na superfície dos pisos é consequiência do calor produzido no interior das camas, em razão da intensa atividade microbiana (Kapuinen, 2001), que pode influenciar a resposta dos suínos, visto que o piso é forma significativa de perda de calor pelos animais (Oliveira et al., 1999).

Não se notou efeito do mês ( $\mathrm{P}>0,05)$, para UR e TSF (Tabela 1), porém, o primeiro mês apresentou a maior média para TA $(\mathrm{P}<0,05)$; para TSC, TMC e T45C, o segundo mês foi superior aos demais $(\mathrm{P}<0,05)$ e, para TMF e T45F as maiores médias foram constatadas nos primeiro e segun- do meses $(\mathrm{P}<0,05)$; entretanto, a TSF não foi alterada nos diferentes meses $(\mathrm{P}>0,05)$.

A UR não variou em função do tempo, nos diferentes meses, mas o efeito dos meses alterou o resultado das demais variáveis. A TA observada no primeiro mês foi superior à dos demais; já as maiores TSC e TMC ocorreram durante o segundo mês, coincidindo com a fase termofílica das camas.

Foi notório o efeito $(\mathrm{P}<0,05)$ da interação entre repetição e mês sobre a UR (Tabela 2), com os maiores valores para as primeira e quarta repetições, ambas no segundo mês; por outro lado, os menores valores dizem respeito à primeira repetição no primeiro mês e para a segunda repetição, no segundo mês, constatando-se efeito da interação entre repetição e mês sobre TA $(P<0,05)$, com a terceira repetição no primeiro mês, apresentando o maior valor mas os menores valores se destinaram às primeira e quarta repetições, em qualquer mês; outrossim, houve efeito da interação entre repetição e mês $(\mathrm{P}<0,05)$, sobre TSC e TMC (Tabela 2). Os maiores e menores valores para essas duas variáveis se deram na interação entre a primeira repetição no segundo mês e para a quarta repetição no terceiro mês, respectivamente. Observou-se efeito da interação entre repetição e mês (P < 0,05 ), sobre TMF, cujos maiores valores foram notados na terceira repetição, nos primeiro e segundo meses, enquanto a menor média se deu na quarta repetição, no terceiro mês. Não ocorreu efeito da interação entre repetição e mês (P > 0,05), sobre as variáveis TMF, T45C e T45F.

Tabela 2. Médias ${ }^{1}$ de umidade relativa do ar (UR\%), temperatura ambiental e das camas $\left({ }^{\circ} \mathrm{C}\right)$ em diferentes locais e profundidades, de acordo com a interação entre repetição $(R)$ e mês $(M)$

\begin{tabular}{|c|c|c|c|c|c|}
\hline$R * M$ & UR & TA & TSC & TMC. & TSF \\
\hline |*1 & $75,0 \mathrm{c}$ & $16,4 \mathrm{~d}$ & $19,7 \mathrm{de}$ & $28,3 f$ & $21,3 \mathrm{~cd}$ \\
\hline $1 * 2$ & 85,4 a & $16,6 \mathrm{~d}$ & $31,0 \mathrm{a}$ & $56,2 \mathrm{a}$ & $22,5 \mathrm{bcd}$ \\
\hline 1*3 & $81,9 a b$ & $14,8 \mathrm{~d}$ & $24,4 b c$ & $37,9 \mathrm{bcd}$ & 20,4 def \\
\hline$\| * 1$ & $83,6 a b$ & $19,2 \mathrm{c}$ & $21,9 \mathrm{~cd}$ & 30,7 ef & $21,4 \mathrm{~cd}$ \\
\hline $11 * 2$ & $74,8 \mathrm{c}$ & $19,3 b c$ & $22,0 \mathrm{~cd}$ & 32,2 def & 21,2 cde \\
\hline $11 * 3$ & $81,0 a b c$ & $21,4 \mathrm{~b}$ & $24,5 b c$ & 29,7 ef & $24,0 \mathrm{bc}$ \\
\hline$\|\mid\| * 1$ & $79,8 a b c$ & 25,3 a & $27,2 \mathrm{~b}$ & $38,6 b c$ & $28,0 \mathrm{a}$ \\
\hline $1 \mid 1 * 2$ & $78,3 \mathrm{bc}$ & $19,6 b c$ & $24,3 b c$ & $43,2 b$ & $25,1 a b$ \\
\hline $111 * 3$ & $80,8 a b c$ & $15,9 d$ & $22,0 \mathrm{~cd}$ & 34,7 cde & $21,4 \mathrm{~cd}$ \\
\hline IN*1 & $83,2 a b$ & $15,9 d$ & 19,7 de & $27,5 f$ & 18,4 ef \\
\hline IN*2 & 85,7 a & $16,1 d$ & $19,5 \mathrm{de}$ & $27,9 f$ & 19,4 def \\
\hline IV*3 & $83,6 \mathrm{ab}$ & $16,5 d$ & $18,0 \mathrm{e}$ & $20,5 \mathrm{~g}$ & $17,7 \mathrm{f}$ \\
\hline
\end{tabular}

${ }^{1}$ Médias seguidas da mesma letra na coluna não diferem significativamente $(\mathrm{P}<0,05)$. UR - umidade relativa do ar, TA - temperatura ambiente, TSC temperatura da cama na superfície, no centro da baia, TMC - temperatura da cama a meia profundidade, no centro da baia, TSF - temperatura da cama na superfície, na frente da baia

Foi grande a variação da UR quando testada a interação entre repetição e mês, haja vista que somente no primeiro mês da primeira repetição e no segundo mês da segunda repetição, os valores estavam abaixo dos $75 \%$ recomendados 
como limite superior, por Veit \& Troutt (1999), para suínos nas fases de crescimento e terminação; os demais valores registrados ultrapassaram este valor. As maiores temperaturas ambientais ocorridas na segunda repetição e nos primeiro e segundo meses da terceira repetição, coincidiram com meses quentes do ano, períodos em que os valores se situaram acima do intervalo de 12 a $21{ }^{\circ} \mathrm{C}$ recomendado por Veit \& Troutt (1999), para suínos em crescimento e terminação e foram superiores, também, ao recomendado por Benedi (2002) de 15 a $18{ }^{\circ} \mathrm{C}$; entretanto, no terceiro mês da primeira repetição, a temperatura média do ar foi inferior a $15^{\circ} \mathrm{C}$, limite recomendado por Benedi (2002). Ainda, segundo Rinaldo (2000), esta variação ao longo dos lotes pode influenciar o conforto térmico dos suínos visto que o fluxo de calor entre os animais e a cama é altamente dependente da temperatura gerada durante a compostagem.

Para as variáveis TSC e TMC na interação entre repetição e mês, os maiores valores ocorreram no segundo mês da primeira repetição e este fato pode ser explicado pela fase termofílica desenvolvida neste período no interior da cama, com liberação de calor para a superfície (Tiquia et al., 1997; Venglovsky et al., 2005); já os menores valores para essas variáveis foram notados no terceiro mês da quarta repetição que, além de ser uma época fria do ano, coincidiu com o final da fase mesofílica, quando a temperatura da cama é um reflexo da temperatura ambiente (Tang et al., 2004).

Não se observou diferença $(P<0,05)$, entre as primeira e segunda camas (Tabela 3) para todas as variáveis; no entanto, a diferença foi significativa para as variáveis TSC, TSF e TMC, com médias superiores para camas novas $(\mathrm{P}<0,05)$ em comparação com camas velhas, embora não se tenha observado diferença $(\mathrm{P}>0,05)$ para UR e TA.

Tabela 3. Médias ${ }^{1}$ de umidade relativa do ar (\%), temperatura ambiental e das camas $\left({ }^{\circ} \mathrm{C}\right)$ em diferentes locais e profundidades

\begin{tabular}{lccccc}
\hline CAMA & UR & TA & TSC & TMC. & TSF \\
\hline PC & $80,2 \mathrm{a}$ & $17,9 \mathrm{a}$ & $23,7 \mathrm{a}$ & $35,3 \mathrm{a}$ & $21,8 \mathrm{a}$ \\
SC & $81,9 \mathrm{a}$ & $18,4 \mathrm{a}$ & $21,9 \mathrm{a}$ & $32,1 \mathrm{a}$ & $21,8 \mathrm{a}$ \\
CN & $80,0 \mathrm{a}$ & $18,3 \mathrm{a}$ & $24,7 \mathrm{a}$ & $39,3 \mathrm{a}$ & $23,2 \mathrm{a}$ \\
CV & $82,1 \mathrm{a}$ & $18,0 \mathrm{a}$ & $20,9 \mathrm{~b}$ & $28,2 \mathrm{~b}$ & $20,3 \mathrm{~b}$ \\
\hline
\end{tabular}

${ }^{1}$ Médias seguidas da mesma letra na coluna não diferem significativamente $(\mathrm{P}<0,05)$. PC - primeiras camas, lotes I e II e SC - segundas camas, lotes III e IV; CN - camas novas, lotes I e III e CV - camas velhas, lotes II e IV. UR umidade relativa do ar, TA- temperatura ambiente, TSC - temperatura da cama na superfície, no centro da baia, TMC temperatura da cama a meia profundidade, no centro da baia, TSF - temperatura da cama na superfície, na frente da baia

Mesmo que tenham sido determinadas em diferentes épocas do ano (Tabela 3), as variáveis UR, TA, TSC e TMC não diferiram entre as PC (primeira e segunda repetições) e SC (terceira e quarta repetições), como previsto, pois a SC foi uma repetição no tempo da PC.

A UR e a TA não diferiram entre camas novas (primeira e terceira repetições), em plena fase termofílica, e camas velhas (segunda e quarta repetições) em fase mesofílica, mas se observou diferença de TSC e TMC, com os maiores valores médios nas camas novas em relação àquelas velhas, o que pode ser atribuído à fase termofílica (Kapuinen, 2001; Tang et al., 2004; Tiquia, 2005).

Durante grande parte do experimento, a UR se situou, em todos os tratamentos, acima dos $70 \%$, ultrapassando o recomendado para suínos nas fases de crescimento e terminação, por Benedi (2002); em situações como esta, de elevada UR, a capacidade dos suínos em dissipar calor por meios evaporativos é diminuída (Morrison et al., 1969). Quanto ao comportamento para a TA, os valores permaneceram, em grande parte do período experimental, dentro da faixa recomendada por Veit \& Troutt (1999) isto é, de 12 a $21{ }^{\circ} \mathrm{C}$ para suínos em crescimento e terminação, mas entre as 16 e $19^{\text {a }}$ semanas e entre as 23 e $32^{\mathrm{a}}$ semanas, os valores da TA ultrapassaram o recomendado; em situação de calor, os suínos podem reduzir sua produção de calor, através do consumo de alimentos, como o ganho de peso (Corrêa et al., 2000; Rinaldo et al., 2000).

Ressalta-se que durante o período experimental a TS, tanto no T1 ou no T2, foi superior ao T3, fato explicado pela atividade microbiana nas camas (Tiquia, 2005; Venglovsky et al., 2005); no T2, embora a cama apresentasse menor profundidade, indicou, também, maior amplitude nas temperaturas observadas na superfície, o que pode influenciar o conforto térmico para os suínos, alterando a perda de calor dos suínos por condução, ao se deitarem no piso (Oliveira et al., 1999). A TMC, tal como para o T1 quanto para o T2, apresentou duas fases termofílicas bem pronunciadas, ou seja, a primeira da 4 à $12^{\mathrm{a}}$ semana, com temperaturas superiores a $60^{\circ} \mathrm{C}$; já na segunda fase termofílica, esta teve duração menor, da 29 a $34^{\mathrm{a}}$ semana, além de uma intensidade menor, não ultrapassando os $60{ }^{\circ} \mathrm{C}$. A TMC foi superior a TA durante o experimento, especialmente durante as fases termofilicas, quando a TMC ultrapassou em mais de $40{ }^{\circ} \mathrm{C}$, a TA, indicando que os processos de compostagem ocorreram de forma adequada (Tiquia et al., 1997; Kapuinen, 2001); entretanto, a TMC não indicou diferença nas variadas alturas de camas.

\section{CONCLUSÃO}

A utilização de piso com cama para suínos nas fases de crescimento-terminação, embora com diferentes profundidades, não foi suficiente para alterar os indicadores de condicionamento ambiental da edificação zootécnica.

\section{LITERATURA CITADA}

Bartels, H. Criação de suíno sobre cama. Agroecologia e Desenvolvimento Rural Sustentável, v.2, n.2, p.16-21, 2001.

Benedi, J. M. H. El Ambiente de los alojamientos ganaderos. 6 ed. Madrid: Ministerio de Agricultura, Pesca y Alimentación, 2002. 28p.

Corrêa, E. K.; Perdomo, C. C.; Jacondino, I. F. Condicionamento ambiental e desempenho de suínos em crescimento e terminação criados sobre piso com leito de cama. Revista Brasileira de Zootecnia, v.29, n.6, p.2072-2079, 2000. 
Fraser, D. The "New perception" of animal agriculture: Legless cows, featherless chickens, and a need for genuine analysis. Journal of Animal Science, v.79, n.4, p.634-641, 2001.

Gentry, J. G.; McGlone J. J.; Blanton Jr. J. R.; Miller M. F. Alternative housing systems for pigs: Influences on growth, composition, and pork quality. Journal of Animal Science, v.80, n.3, p.1781-1790, 2002.

Honeyman, M. S. Sustainability Issues of U.S. in swine production. Journal of Animal Science, v.74, n.2, p.1410-1417, 1996.

Honeyman, M. S. Extensive bedded indoor and outdoor pig production systems in USA: Current trends and effects on animal care and product quality. Livestock Production Science, v.94, n.6, p.15-24, 2005.

Honeyman M. S; Harmon. J. D. Performance of finishing pigs in hoop structures and confinement during winter and summer. Journal of Animal Science, v.81, n.5, p.1663-1670, 2003.

Jongbloed A. W; Lenis N. P. Environmental concerns about animal manure. Journal of Animal Science, v.76, n.3, p.2641-2648, 1998.

Kapuinen, P. Deep litter systems for beef cattle housed in uninsulated barns, Part 2: temperature and nutrients. Journal Agricultural Research, v.80, n.1, p.87-97, 2001.

Morrison, S. R.; Heitman, H.; Bond, T. E. Effect of humidity on swine at temperatures above optimum. International Journal of Biometeorology, v.13, n.4, p.135-139, 1969.

NRC - National Research Council. Nutrient requeriments of swine. 9.ed. Washington: The National Academies Press 1998. 93p.
Oliveira, J. A.; Meunier-Salaüm, M. C.; Robin, P.; Tonnel, N.; Fraboule, J.B. Analyse du comportement du porc en engraissement eleve sur litiére de sciure ou sur caillebotis integral. Journées de Recherche Porcine en France, v.31, n.4, p.117-123, 1999.

Rinaldo, D.; Dividich J.; Nobletb, J. Adverse effects of tropical climate on voluntary feed intake and performance of growing pigs. Livestock Production Science, v.66, n.3, p.223-234, 2000.

Statistix. Statistix ${ }^{\circledR} 8$ analytical software. Tallahassee: Analytical Sofware, 2003.

Tang, J. C.; Kanamori, T.; Inoue, Y. Changes in the microbial community structure during thermophilic composting of manure as detected by quinone profile method. Process Biochemistry, v.39, n.2, p.1999-2006, 2004.

Tiquia, S. M. Microbiological parameters as indicators of compost maturity. Journal of Applied Microbiology, v.99, n.4, p. 816-828, 2005.

Tiquia, S. M.; Tam, N. F. Y.; Hodgkiss, I. J. Effects of turning frequency on composting of spent pig-manure sawdust litter. Bioresource Technology, v.62, n.2, p.37-42, 1997.

Veit, H. P.; Troutt, H. F. Monitoring air quality for livestock respiratory health. Veterinary Medicine and Small Animal Clinician, v.77, n.1, p.454-464, 1999.

Venglovsky, J.; Sasakova N.; Vargova M.; Pacajova Z.; Placha I.; Petrovsky M.; Harichova D. Evolution of temperature and chemical parameters during composting of the pig slurry solid fraction amended with natural zeolite. Bioresource Technology, v.96, n.2, p.181-199, 2005.

Wang, P.; Changa, C. M.; Waston, M. E.; Dick. Maturity indices for composted dairy and pig manures. Soil Biology \& Biochemistry, v.36, n.4, p.767-776, 2004. 